\title{
Application of Biomaterials and Inkjet Printing to Develop Bacterial Culture System
}

\author{
Tithimanan Srimongkon, ${ }^{1}$ Shusaku Mandai, ${ }^{2}$ and Toshiharu Enomae ${ }^{1}$ \\ ${ }^{1}$ Graduate School of Life and Environmental Sciences, University of Tsukuba, Tsukuba 305-8572, Japan \\ ${ }^{2}$ Nippon Synthetic Chemical Industry Co., Ltd., Kyoto 567-0052, Japan \\ Correspondence should be addressed to Tithimanan Srimongkon; s1230253@u.tsukuba.ac.jp
}

Received 13 March 2015; Revised 13 June 2015; Accepted 13 July 2015

Academic Editor: Ying Liu

Copyright (c) 2015 Tithimanan Srimongkon et al. This is an open access article distributed under the Creative Commons Attribution License, which permits unrestricted use, distribution, and reproduction in any medium, provided the original work is properly cited.

\begin{abstract}
We created an automated bioassay system based on inkjet printing. Compared to conventional manual bacterial culture systems our printing approach improves the quality as well as the processing speed. A hydrophobic/hydrophilic pattern as a container supporting a culture medium was built on filter paper using a toluene solution of polystyrene for hydrophobization, followed by toluene printing to create several hydrophilic areas. As culture media we used a novel poly(vinyl alcohol) based hydrogel and a standard calcium alginate hydrogel. The poly(vinyl alcohol) hydrogel was formed by physical crosslinking poly(vinyl alcohol) with adipic acid dihydrazide solutions. The conditions of poly(vinyl alcohol) gelation were optimized for inkjet printability and the optimum mixture ratio was determined. The calcium alginate hydrogel was formed by chemical reaction between sodium alginate and $\mathrm{CaCl}_{2}$ solutions. Together with nutrients both hydrogel solutions were successfully printed on paper by means of the modified inkjet printer. The amount of each solution was demanded simply by outputting CMYK values. In the last step bacterial cells were printed on both hydrogel media. For both media we achieved a stable bacteria growth which was confirmed by microscopical imaging of the developed bacterial colonies.
\end{abstract}

\section{Introduction}

Inkjet-based bioprinting becomes increasingly important for scientific and industrial use related to bio- and biomedical applications. One of its main advantages is that it combines resource saving with noncontact processing attained by the drop on demand (DOD) system [1]. The possibility of accurately dispensing very small quantities of fluids on substrates made it a versatile tool for a broad range of applications such as 3D printing of microscopic bacterial communities using a laser-based lithographic technique [2], heterogeneous tissue construction [3], protein and cell printing [4], organ 3D printing design [5-7], tissue engineering and development $[8,9]$, cell molecular self-assembly [10], DNA [11-13], hybrid cells [14], and membranes [15]. Moreover, the hazards caused by careless handling of pathogenic bacteria can be minimized by inkjet printing, as it is possible to dispense the minimum required amount of a bacterial suspension.

In this study, two different types of commercial inkjet printers were used as dispensing devices in a comprehensive bacterial culture system. Conventionally, bacterial culture systems utilize agarose gels containing nutrients as the medium. Agarose is a galactan formed by linking approximately 500 agarobioses consisting of D-galactose and 3,6anhydro-L-galactopyranose linked by $\alpha-(1 \rightarrow 3)$ and $\beta-(1 \rightarrow$ 4) glycosidic bonds. In a previous study [16], we utilized an inkjet printer to dispense both agarose medium and a bacterial suspension on a paper substrate, with the goal of creating stable patterns and dispensing accurate amounts of bacterial cells. Although being in principle suitable for printing agarose-based gels suffer from two major drawbacks. Common agarose solution has very high viscosity and as agarose contains long chains of polysaccharides and solidifies after cooling, the temperature must be maintained higher than $37^{\circ} \mathrm{C}$ to prevent gelling inside the printing system. Both make it unsuitable for smooth ejection from the head nozzles of the inkjet printer. Therefore, agarose was hydrolyzed by using sulfuric acid to reduce the degree of polymerization. After hydrolysis, the agarose became acidic and was thought 


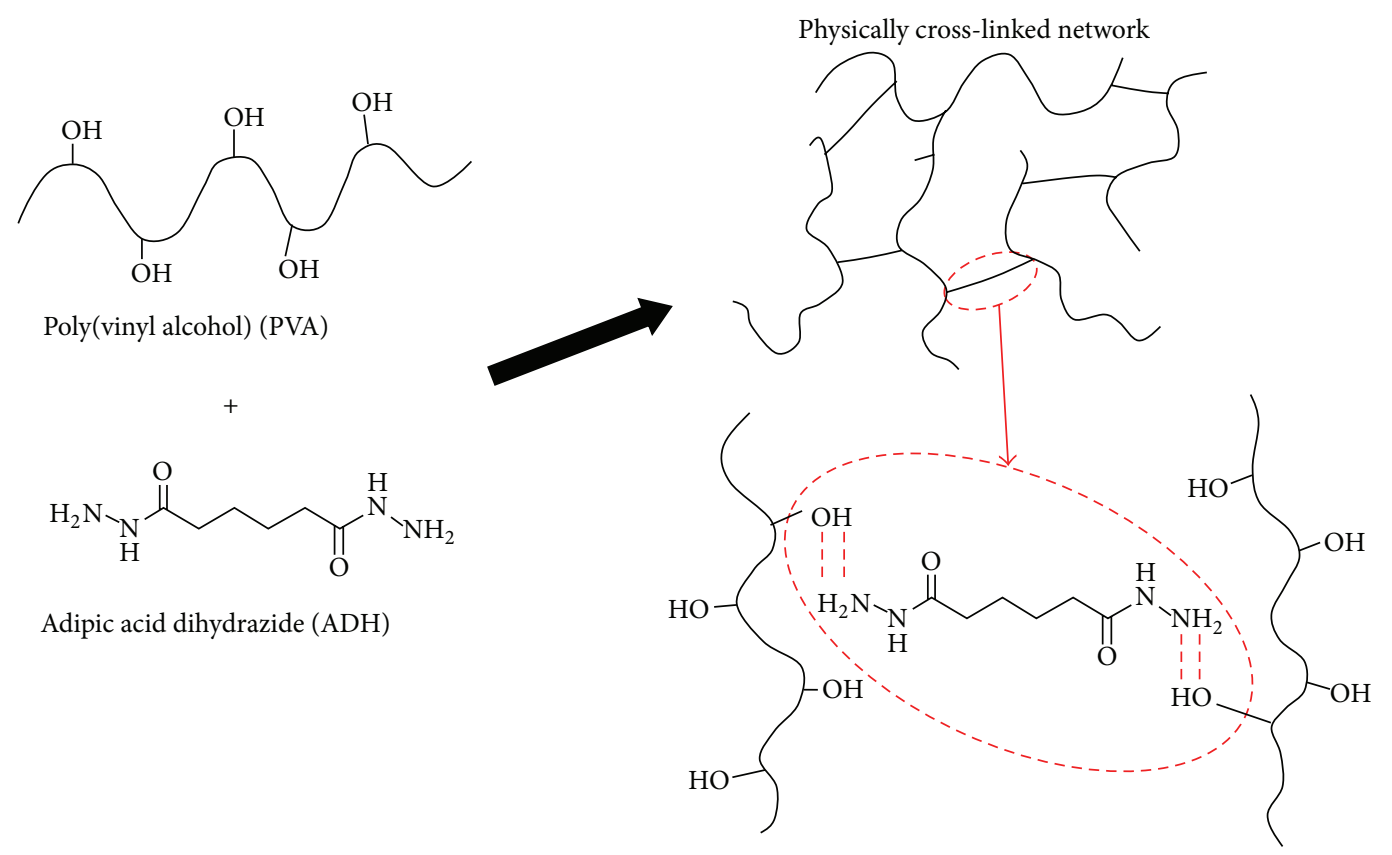

FIGURE 1: Formation of a PVA hydrogel network based on intermolecular hydrogen bonding.

to inhibit bacterial growth. To avoid this problem, the $\mathrm{pH}$ was adjusted to neutral using sodium hydroxide, and the hydrolyzed agarose was successfully ejected by the inkjet printer. However, the hydrolysis processes took a long time, and the viscosity adjustment required precise control. To overcome this shortcomings we introduce in this work two culture media, poly(vinyl alcohol) (PVA) hydrogel and calcium alginate (CA) hydrogel, as alternative to agarose. Both are shown to be very well suited for printing, allowing smoother and more efficient operation of the culture system.

Hydrogels are formed by cross-linking polymer chains through physical, ionic, or covalent interactions and are well known for their ability to absorb water [17-19]. They are widely used for various pharmaceutical and biomedical applications [20-23].

PVA is a polymer that can be used to form hydrogels. PVA can be cross-linked with many types of cross-linking agents [24]. In this study, adipic acid dihydrazide (ADH) was mixed with PVA as a cross-linking agent to produce PVA hydrogels. The PVA hydrogel is classified as a reversible or physical gel. PVA hydrogel networks are held together by hydrogen bonding as shown in Figure 1.

In physically cross-linked gels, dissolution is prevented by physical interactions between different polymer chains [25]. All such physical interactions are reversible and can be disrupted by changes in physical conditions or by application of stress [26]. We observed that PVA hydrogel reverted to a PVA/ADH mixture solution after several days under ambient conditions. The gelation behavior of a PVA hydrogel depends on the concentration of PVA, the ratio of PVA and ADH in the mixture, and the mixing intensity; all of these parameters are closely related to the ejection from an inkjet printer.
Alginic acid or alginate, which is an anionic polysaccharide consisting of homopolymeric blocks of $\beta$-D-mannuronate and $\alpha$-L-guluronate residues derived from cell walls of brown algae, forms a gel in the presence of calcium and dissolves to a solution upon addition of a calcium chelating agent.

Calcium ions as a crosslinking agent diffuse from a bulk solution into flocculated sodium alginate (SA) [27]. The hydrogel forms by chemical reaction; the calcium ions displace sodium ions from the alginate, holding the long alginate molecules together such that a gel formed (Figure 2). Unlike agarose, the PVA/ADH mixture and the $\mathrm{SA} / \mathrm{CaCl}_{2}$ components remain under ambient condition long enough in a solution state to be processed by printing. Several studies pointed out the benefits of sodium alginate to bacterial cells and tissue culture, such as increasing the survival of bacteria [28], enhancing the bacterial yield [29], and proliferation [30].

In this research, PVA and CA hydrogels were used as the culture media and printed on paper by using inkjet printer. Subsequently, properties of PVA and CA hydrogels were compared in terms of the operation performance and the applicability of those gels was evaluated as culture media on paper.

\section{Materials and Methods}

2.1. Preparation of Paper Substrates. Because conventional filter paper is too water absorbent to hold a PVA hydrogel solution near the surface firmly, it is necessary to locally increase the hydrophobization of the paper substrate. In a previous work, we introduced a method to modify paper 


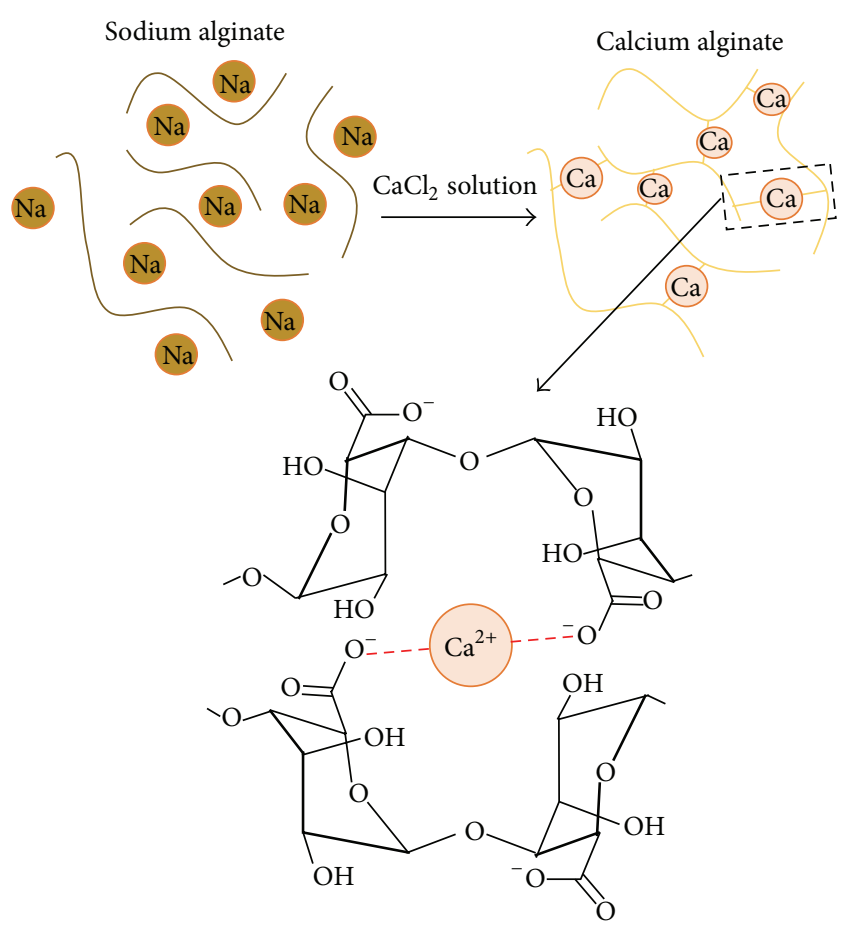

Figure 2: Reaction of $\mathrm{SA}$ and $\mathrm{CaCl}_{2}$.

to form hydrophobic and hydrophilic areas on the same substrate by using polystyrene (PS) and toluene [16]. The filter paper was soaked in a $3.0 \mathrm{wt} \%$ toluene solution of PS for $1 \mathrm{~h}$ and then allowed to dry at room temperature for $15 \mathrm{~min}$ to hydrophobize the entire filter paper. Squares $(10 \mathrm{~mm} \times$ $10 \mathrm{~mm}$ ) were etched to make hydrophilic areas by printing toluene with 3 head nozzles of the SCS inkjet printer as referred to hereinafter and overprinting the area twice for the best organized patterns with distinctive borders. Finally, hydrophilicity was tested by dropping $2 \mu \mathrm{L}$ of water on the etched areas, and until the time required for the water drop to penetrate and disappear from the surface reaches about $10 \mathrm{~s}$, the degree of etching was adjusted.

2.2. Preparation of PVA Hydrogel Ink. First, PVA hydrogels were prepared by mixing PVA (Gohzenol Z-100, Nippon Gohsei, Japan) and ADH (Adipic acid dihydrazide, Nippon Kasei Chemical, Co., Ltd., Japan) solutions at several concentrations and a variety of different mixture ratios. The viscosities of the PVA solutions were then examined with a glass capillary viscometer (Cannon-Fenske type) in a water bath (Kinematic Viscosity Bath TV-5s, Japan) to evaluate the suitability of the hydrogel for inkjet printing. In practical printing, an ink cartridge is filled with a mixture of PVA and $\mathrm{ADH}$ before gelling. Next, an $\mathrm{ADH}$ solution was poured into a PVA solution, and the mixture was stirred for $5 \mathrm{~min}$.

We defined the gelation of PVA hydrogel by gel strength (rigidity modulus). The gel strength was measured using a rheometer (CR-500DX, Sun Scientific Co., Ltd, Japan) under the following conditions: $60 \mathrm{~mm} / \mathrm{min}$ intrusion velocity with a plunger having a pressure-sensing head of $10 \mathrm{~mm}$ in diameter and $10 \mathrm{~mm}$ in full depth. The force sensed by the sensing head was recorded over the full depth of $10 \mathrm{~mm}$ and the result was reported as the final force in $\mathrm{N}$ at $10 \mathrm{~mm}$ in depth and plotted on a ternary phase diagram. The rheometer was also used to characterize the gelation time [25] by measuring the gel strength every 5 min starting from the initial preparation of the mixture until a finite gel strength was observed. After determination of the mixture ratio suitable for gel formation (in terms of gel strength, as evaluated using the ternary phase diagram), the gelation times of the PVA/ADH mixtures were measured.

\subsection{Ultraviolet (UV) Absorption Spectra of PVA Hydrogel.} The PVA/ADH mixture in a ratio of 4.5:0.5:95 (PVA: $\mathrm{ADH}: \mathrm{H}_{2} \mathrm{O}$ ) was investigated by ultraviolet spectroscopy. The investigated wavelength range was from $200 \mathrm{~nm}$ to $300 \mathrm{~nm}$ with a step size of $20 \mathrm{~nm}$ using an ultraviolet spectrophotometer (UV-3100PC, Shimadzu Scientific Instruments, Japan). The PVA/ADH mixture was measured immediately, $1 \mathrm{~min}$, $10 \mathrm{~min}, 15 \mathrm{~min}$, and $20 \mathrm{~min}$ after mixing to monitor the transitional condition of PVA hydrogel formation with time. For comparison, we also measured the UV absorption spectrum of PVA solution and ADH solution (both at $0.05 \%$ ).

2.4. Preparation of CA Hydrogel Ink. Usually, media are prepared by mixing a SA solution, $0.05 \%$ (w/w) of $\mathrm{CaCl}_{2}$ solution, $0.05 \mathrm{~g} / \mathrm{L}$ of yeast extract solution, and $0.1 \mathrm{~g} / \mathrm{L}$ of bacto tryptone solution in required ratios, where the unit $\mathrm{g} / \mathrm{L}$ means a solute in $\mathrm{g}$ dissolved in $1 \mathrm{~L}$ of an aqueous solution. The ratio was controlled based on the amount ejected from each cartridge. However, the inkjet ejection performance is mainly determined by the ink viscosity [16]. In particular, SA solution has a higher viscosity than the other solutions and its viscosity can be controlled by the SA concentration. Therefore, the SA solutions at several concentrations $(0.01 \%$, $0.025 \%, 0.05 \%$, and $0.1 \%(\mathrm{w} / \mathrm{w}))$ were prepared and their viscosities were measured with a glass capillary viscometer. The gelation of CA hydrogel was formed by an irreversible reaction. However, in order to provide CA hydrogel without remaining initial substances, the ratio of $\mathrm{SA}$ and $\mathrm{CaCl}_{2}$ solutions was examined by measuring $\mathrm{pH}$ values. In the experiment, the $\mathrm{pH}$ values of $\mathrm{SA}$ and $\mathrm{CaCl}_{2}$, solutions were 8.0 and 7.0, respectively. After CA hydrogel was formed, the $\mathrm{pH}$ value of a remaining solution was examined. Then the ratio without remaining initial substances was adjusted.

2.5. Culture Media Printing. We used 2 different kinds of inkjet printers as dispensing tools, namely, a single cartridge system (SCS) (Dimatix DMP-2831, Fujifilm, Japan) and a multicartridge system (MCS) (iP2000, Canon, Japan). Subsequently, the efficiency of the 2 systems was compared in terms of the compatibility with the hydrogel culture media and length of time required for one complete printing operation.

2.5.1. SCS Printer. As the SCS printer allows setting only one cartridge, all solutions were loaded into separate cartridges and printed one by one, respectively. The methods for 

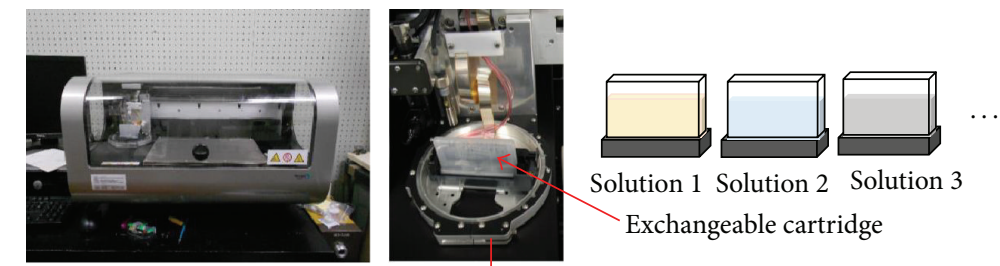

Solution 1 Solution 2 Solution 3

Exchangeable cartridge

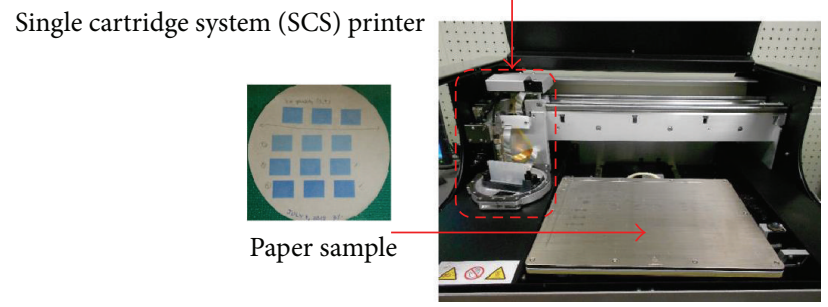

FIGURE 3: Medium printing by SCS printer sequence.

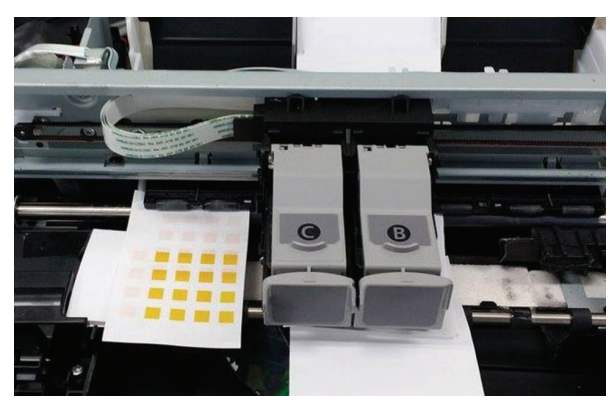

FIgURE 4: Modified printer with dummy paper.

the SCS printer are shown in Figure 3. The printing condition was $40 \mathrm{~V}$ in ejection voltage, 16 head nozzles in use, $50 \mu \mathrm{m}$ in drop spacing, and at least 10 times overprinting.

2.5.2. MCS Printer. Overprinting is required in order to obtain a culture medium with sufficient thickness. However, reloading a paper sample with the culture medium already printed was not successful because the printed media was smeared by feeding rollers of the printer during printing. Therefore, for continuous overprinting without paper reloading, the printer was modified by perforation. After both the upper and lower parts of the printer stage were perforated so that a culture medium would be printed on a static paper sample set on the far left side of the printing area while dummy paper would be loaded onto the paper tray smoothly as shown in Figure 4.

To load hydrogel medium materials, the sponge in the ink cartridge was removed and the cartridge was washed with distilled water. To sterilize the cartridge for subsequent bacterial culture tests, the cartridge was sprayed with aqueous ethanol (70\%).

The MCS printer for color printing can be equipped with up to 4 different cartridges loaded with 4 different kinds of inks so that all solutions composing a hydrogel medium could be printed at the same time. In addition, the ejected amount of each solution was controlled by specifying CMYK percentages. The photographs of the modified MCS printer are shown in Figure 5.

2.6. Bacterial Culture. In our previous work [16], Escherichia coli (E. coli) was tested as an example of bacteria for the printability on a SCS printer. It could be printed successfully and a high survival ratio was obtained. Therefore, in this work, bacteria were taken randomly from a stationery surface and isolated for pure culture by the streak plate technique. Then, E. coli was recognized by the morphology of the bacterial colonies. The concentration of the bacterial suspension was diluted to an aqueous suspension of $2.7 \times 10^{7}$ colonyforming units $(\mathrm{CFU}) / \mathrm{mL}$ using the serial dilution method. Then, $0.5 \mathrm{~mL}$ of the diluted bacteria was mixed with $1 \mathrm{~mL}$ of glycerol and loaded into a cartridge of the SCS printer. First, we printed 16 areas of PVA hydrogel and CA, respectively, on a sheet of hydrophobic/hydrophilic paper. Next, the bacterial suspension was printed on the top of the PVA hydrogel and CA hydrogel media. In total, we prepared 5 sheets containing 16 areas of bacteria. After $24 \mathrm{~h}$, the average number of grown colonies per area was determined by optical microscopy. The survival rate is defined as the proportion of the number of bacterial growth areas to the total number of areas. In the same way, the contamination rate was defined as the proportion of the number of contaminated areas to the total number of areas.

\section{Results and Discussion}

3.1. Characteristics and Optimization of PVA Hydrogel. Figure 6 shows 2 examples of the force curves used to determine the gel strengths of the hydrogels. The sensed force attained a maximum value at a depth of $10 \mathrm{~mm}$ for every curve. The gel strengths (maximum force values) are plotted in the ternary phase diagram as shown in Figure 7 as a function of dry mass percentages of PVA and $\mathrm{ADH}$, with water content in percentage of the mixture. The ratios of the three components comprising the gel were distributed as shown in the shaded area in Figure 7. Some PVA/ADH mixtures gelled by themselves and had various gel strengths. No force 


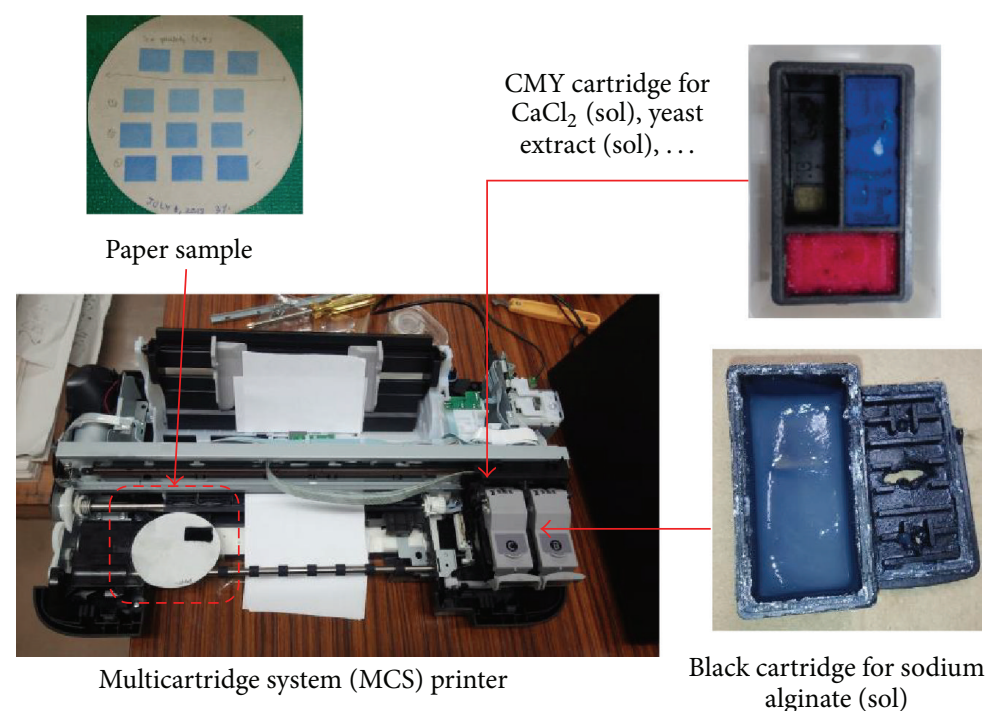

FIGURE 5: Processing of MCS printer and procedure of medium printing.

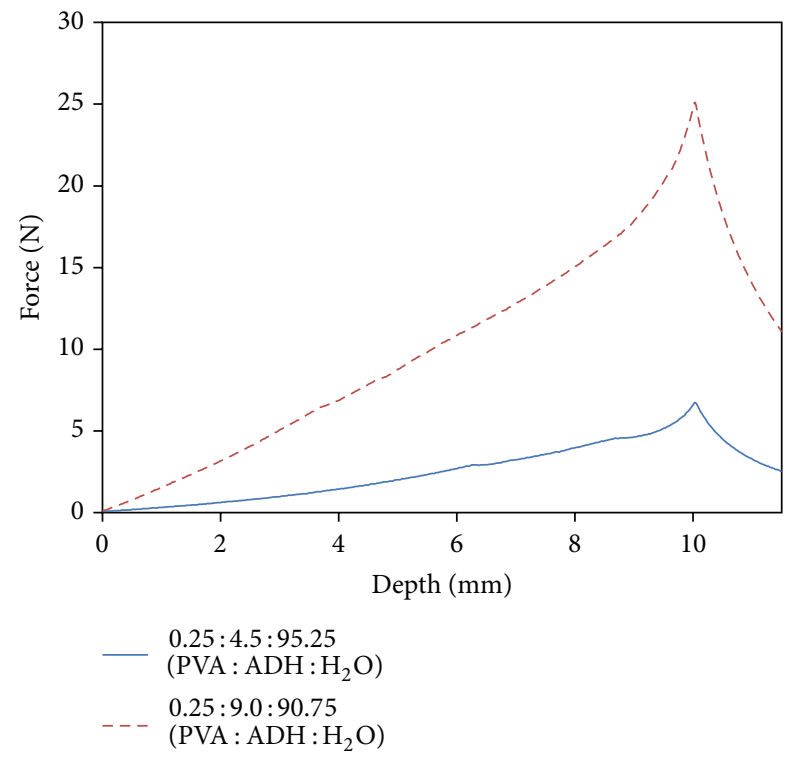

FIGURE 6: Force curves of the cylindrical plunger sensor in intruding PVA hydrogel over time down to $10 \mathrm{~mm}$ in depth.

(practically less than $1 \mathrm{~N}$ ) was detected for the others (no hydrogel formation identified). The gel strength depended mainly on the dry mass percentages of PVA. However, when the concentration of $\mathrm{ADH}$ exceeds $0.75 \%$ dry mass, gelation was prevented for any ratio of $\mathrm{PVA} / \mathrm{ADH} / \mathrm{H}_{2} \mathrm{O}$ mixture. Also the percentage of PVA was reduced to less than $4.50 \%$; hydrogel could not form. In contrast, $25 \%$ or more PVA produced hydrogel without the addition of a cross-linking agent and cannot be handled as an ink.

Increasing the dry mass percentages of PVA also affected the gelation time, as shown in Figure 8. To identify the optimal concentrations of PVA and $\mathrm{ADH}$ suitable for inkjet

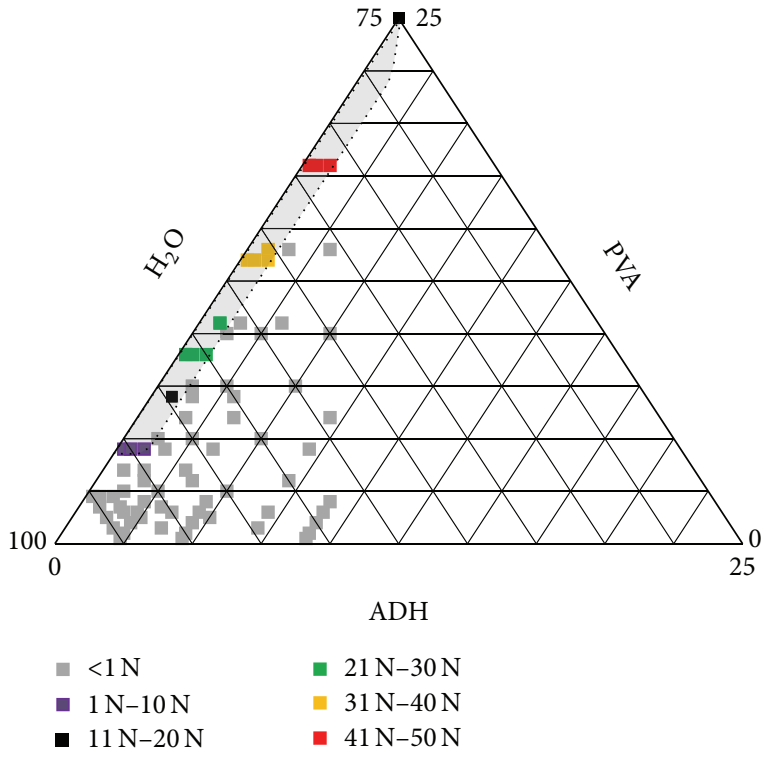

FIgURE 7: Ternary phase diagram of gel strength as a function of the ratios of the 3 components composing PVA hydrogel and ratios of gelation were observed in the shaded area.

printing, we examined all PVA/ADH mixtures in the shaded area in the ternary phase diagram with respect to their printing capabilities. The mixture has to be chosen such that it gels as fast as possible on paper while also remaining stable in solution long enough to be stored in the printer cartridge during the preparation process. Consequently, we identified 4.5\% dry mass PVA and $0.5 \% \mathrm{ADH}$ as the best combination with respect to printability and quality of the printed patterns. Moreover, the determined optimal mixture also allowed the moisture to be maintained for at least 2 days which was long enough for the rapid bacterial culture system. It has to be noted that even at PVA dry mass percentages higher than 


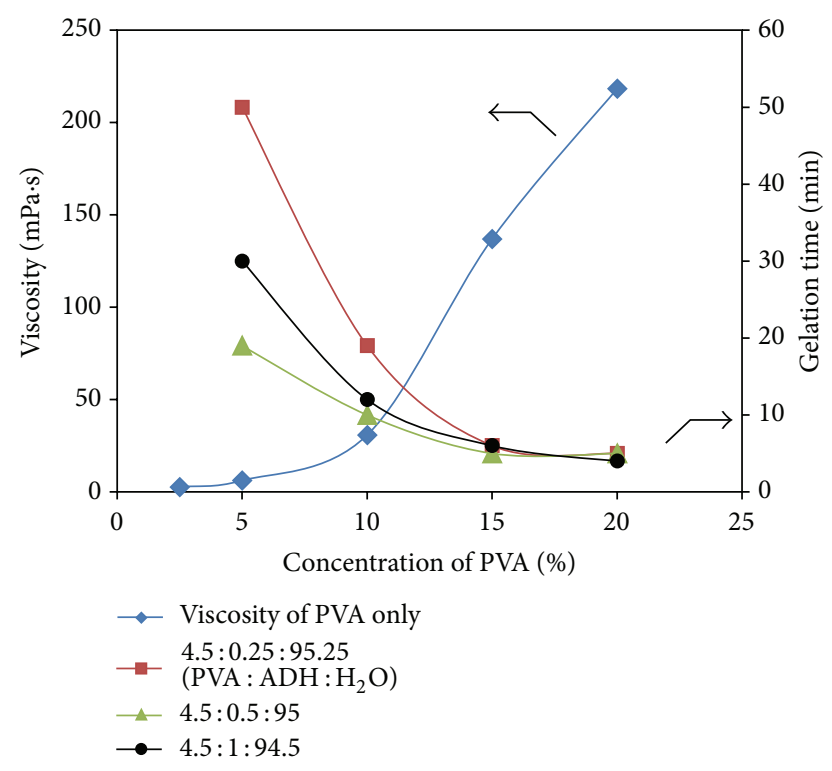

FIGURE 8: Viscosity of PVA solutions and gelation time of the PVA hydrogel at three concentrations (PVA : $\mathrm{ADH}: \mathrm{H}_{2} \mathrm{O}$ ).

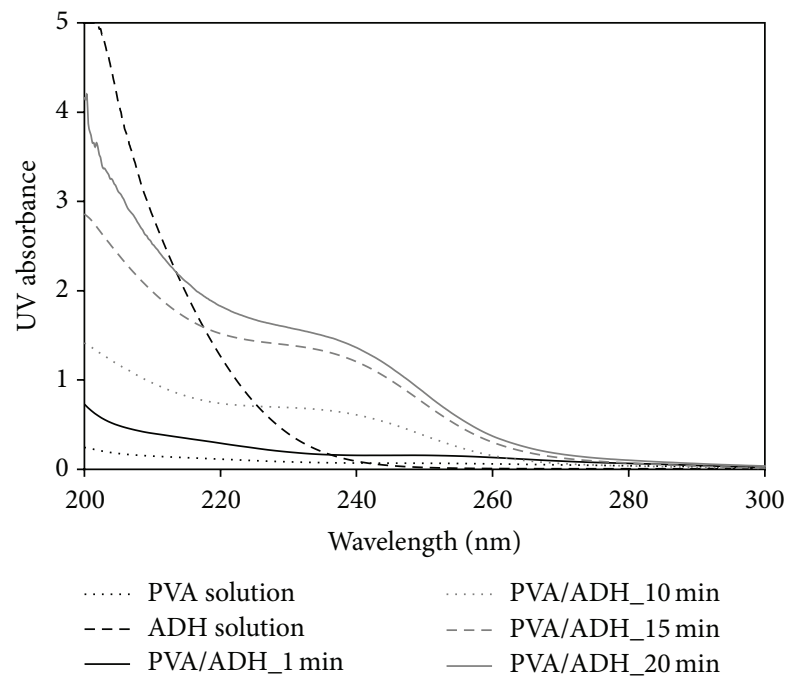

FIgURE 9: UV absorbance of ADH, PVA, and the PVA/ADH mixtures with increasing degrees of gelation at 1, 10, 15, and 20 min (full gelation).

$13.5 \%$, the mixture gelled with an appropriate gelation time; however, the viscosity was too high for inkjet printing.

Figure 9 shows the UV absorption spectra of the PVA solution, ADH solution, and PVA/ADH mixtures. Four spectra of the mixtures were measured at each stage of the transition from solution to full gelation for the identical sample. Some studies have shown that hydrogen bonding can affect UV absorption [31, 32]. In the UV spectra, a shoulder peak appeared for the PVA/ADH mixture around $240 \mathrm{~nm}$ and could be assigned to the hydrogel structure. The shoulder peak height continued to increase with time, suggesting continuous gelation. Between $15 \mathrm{~min}$ and $20 \mathrm{~min}$ in elapsed

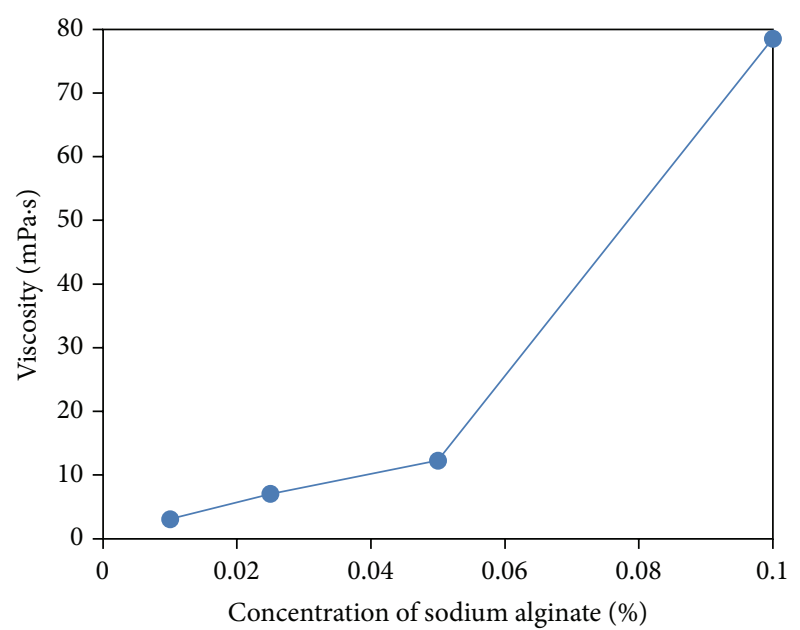

FIGURE 10: Viscosity of SA solutions at various concentrations.

time, the difference in the spectra was slight. This behavior is likely to correspond to the approach to the complete gelation. Although the curve of the $\mathrm{ADH}$ solution exhibited much higher UV absorption in the shorter wavelength band than PVA at the same concentration, there was no clear peak for quantifying $\mathrm{ADH}$ independent of the hydrogel.

3.2. CA Hydrogel Medium. The viscosity of the sodium alginate solutions was examined for an optimum viscosity level for the printers. The determined dependence of the viscosity on the concentration is shown in Figure 10.

3.3. Culture Media Printing. Media printing was tested by printing all solutions using both the SCS and MCS printers to evaluate the compatibility between the printers and solutions. The result in Table 1 shows that the maximum concentration which could be printed by the SCS printer is only $2.25 \%$ dry mass of PVA in PVA/ADH mixture. For the multicartridge MCS printer the range of printable mixture ratios is much wider. By means of the MCS printer we could successfully print $2.25 \%, 4.5 \%$, and $9 \%$ dry mass of PVA in PVA/ADH mixtures. We found that the SCS printer was not suitable for PVA/ADH mixture printing. As previously described in Characteristics and Optimization of PVA Hydrogel, $4.5 \%$ dry mass PVA and $0.5 \% \mathrm{ADH}$ with the MCS printer was identified as the best combination. It combines adequately low viscosity which ensures continuous ejection with no clogging and a relatively large amount of PVA per droplet for rapid gelling. Additionally, this mixture maintained stability in the solution state long enough to be stored in an ink cartridge.

Regarding CA hydrogel, the result in Table 1 shows that all components could be printed by SCS and MCS printers. High concentrations of SA solutions could not be printed by SCS. Considering the viscosities of PVA and SA solutions, SCS is likely to allow printing inks with viscosities of lower than 10 $\mathrm{mPa}$.s. A $0.025 \%(\mathrm{w} / \mathrm{v})$ of SA solution was chosen as the ink for loading. Other solutions for making CA hydrogel were 


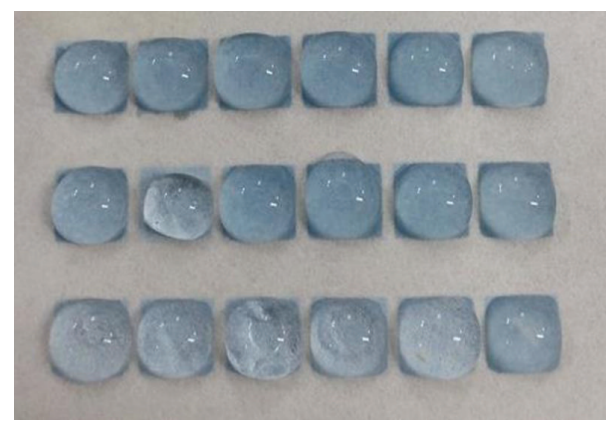

FIGURE 11: CA hydrogel on hydrophilic areas on hydrophilic/hydrophobic patterned paper.

TABLE 1: Printability and time required per sample of SCS and MCS printers with sodium alginate (SA) solutions.

\begin{tabular}{lcc}
\hline & SCS & MCS \\
\hline Printability of solutions & & \\
PVA/ADH/ ${ }_{2} \mathrm{O}$ mixture & & \\
$2.25: 0.5: 97.25$ & $\boldsymbol{x}$ & $\checkmark$ \\
$4.5: 0.5: 95$ & $\boldsymbol{x}$ & $\checkmark$ \\
$9: 0.5: 90.5$ & $\boldsymbol{x}$ & $\checkmark$ \\
$13.5: 0.5: 86$ & $\boldsymbol{x}$ & $\boldsymbol{x}$ \\
$18: 0.5: 81.5$ & $\checkmark$ & $\checkmark$ \\
SA $0.01 \%$ & $\checkmark$ & $\checkmark$ \\
SA $0.025 \%$ & $\boldsymbol{x}$ & $\checkmark$ \\
SA $0.05 \%$ & $\boldsymbol{x}$ & $\checkmark$ \\
SA $0.1 \%$ & $\checkmark$ & $\checkmark$ \\
CaCl ${ }_{2} 0.05 \%$ & $\checkmark$ & $\checkmark$ \\
Bacto tryptone $0.1 \mathrm{~g} / \mathrm{L}$ & $\checkmark$ & $\checkmark$ \\
Yeast extract $0.05 \mathrm{~g} / \mathrm{L}$ & & \\
Time required per sample & 120 & 40 \\
Average (min) & 0.00 & 0.44 \\
Accuracy error (\%) &
\end{tabular}

$\checkmark$ : successfully printed; $\boldsymbol{X}$ : unable to eject.

found to be all printable. To adjust the mixture ratio of all the components to follow the standard CA medium composition such as a suitable mass ratio of $5: 6\left(\mathrm{SA}: \mathrm{CaCl}_{2}\right)$, the ejection percentage of each color cartridge called CMYK for MCS was decided to be $50 \%$ of $\mathrm{SA}, 30 \%$ of $\mathrm{CaCl}_{2}, 15 \%$ of Bacto tryptone, and $5 \%$ of yeast extract. The CA hydrogel media printed on a filter paper substrate is shown in Figure 11.

Using a camera installed near the head nozzles we found that the SCS printer has a high accuracy in the positioning of the head nozzles which is important for overprinting the solution on the paper. However, the MCS printer is less time consuming because all solutions are printed at the same time and, among others, the composition of all inks can be altered very easily by changing CMYK percentages from area to area on the same paper substrate.

3.4. Bacterial Growth on the PVA Hydrogel and CA Hydrogel Media. For both PVA hydrogel medium and CA hydrogel medium the $E$. coli growth on the paper was confirmed $24 \mathrm{~h}$
TABLE 2: Survival rate of E. coli on PVA and CA hydrogel media after $24 \mathrm{~h}$.

\begin{tabular}{lcc}
\hline & PVA hydrogel & CA hydrogel \\
\hline $\begin{array}{l}\text { Number of areas measured } \\
\begin{array}{l}\text { Mean number of colonies per } \\
\text { area }\end{array}\end{array}$ & 60 & 80 \\
$\begin{array}{l}\text { 95\% confidence intervals of } \\
\text { number of colonies per area }\end{array}$ & 0.27 & 5.73 \\
$\begin{array}{l}\text { Survival rate (\%) } \\
\text { Contamination rate (\%) }\end{array}$ & 67.1 & 0.22 \\
\hline
\end{tabular}

after inoculation. The counted number of colonies is listed in Table 2 . The average number (95\% confidence intervals) of colonies per hydrophilic area was found to be comparable for both hydrogels, with a slightly higher number for PVA hydrogel. This low deviation suggests that liquids containing E. coli cells could be dispensed evenly and regularly onto a culture medium. E. coli cells on each media are shown in Figure 12.

The results showed that PVA and CA hydrogels could be printed by using SCS and MCS printers on paper. In addition, living E. coli cells could be printed and survived the printing process. SA is a polysaccharide which is indigestible for most organisms so that microbial growth does not affect the gel used, meaning that CA hydrogel media maintain the stable quality [29]. The survival rate of the CA hydrogel was higher than that of the PVA hydrogel which exhibits an acidic $\mathrm{pH}$ $[17,18]$. In our experiment, the $\mathrm{pH}$ of the PVA hydrogel was 6.0 and for the CA hydrogel the $\mathrm{pH}$ was 7.0.

Both printing systems have their own advantages and can be used complementary depending on the desired application. The results showed that the SCS printer has higher accuracy in positioning the printing location and dispensing stable amounts of inks. On the other hand, the MCS printer is less time consuming and it is easier to achieve various compositions of medium components by means of simple CMYK color adjustment. Summarizing, the system we developed in the present work provides a low-cost, easyto-use, efficient means for bacterial culture. Essentially we modified an inkjet printer to function as a dispensing device and clean bench and paper were used as a container for the culture media. An actual productive unit based on the techniques developed can be realized as sketched in Figure 13 which shows the possible schematics of a future system for efficient bioassay.

\section{Conclusion}

In this study, we combined inkjet printing and paper technologies for creating a prototype of an automated and simple to operate bacterial culture system. PVA hydrogel, formed by spontaneous physical aggregation of PVA and $\mathrm{ADH}$ aqueous solutions, was applied as a culture medium. In the same way, CA hydrogel formed by chemical reaction between SA solution and $\mathrm{CaCl}_{2}$ solution. We used two different kind of printers, namely, a single cartridge SCS printer and a multicartridge MCS printer to dispense the culture media 


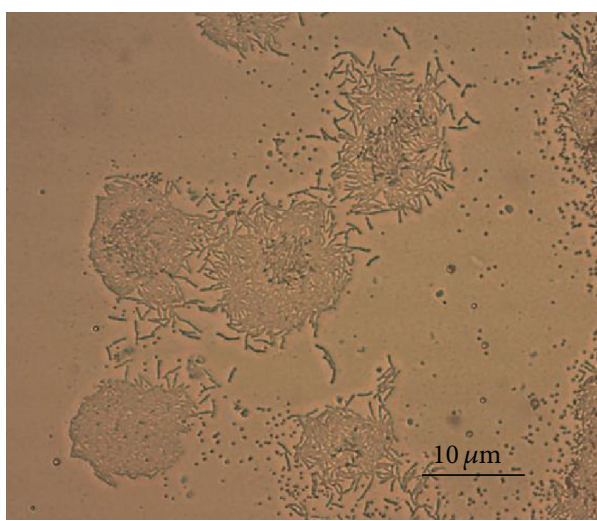

(a)

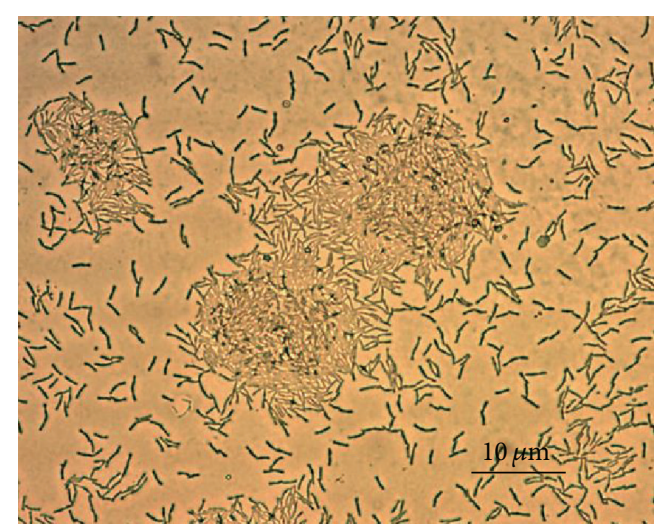

(b)

FIGURE 12: E. coli colonies growing on PVA hydrogel medium (a) and SA medium (b) after $6 \mathrm{~h}$.

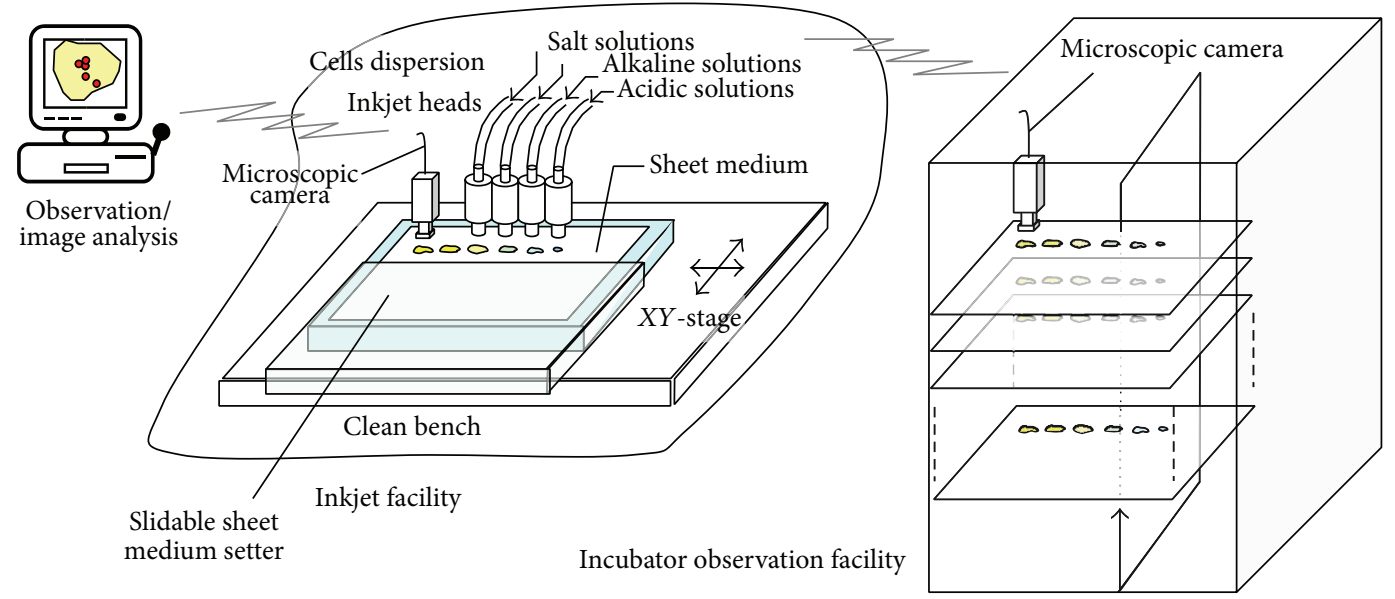

FIGURE 13: Bioassay system using inkjet printing and paper substrate.

and bacterial suspension. The SCS printer showed on the expense of processing speed a high accuracy in positioning of the printing location and provided an accurate overprinting. On the other hand, the MCS printer had a larger margin of accuracy error in the time sequence; however, it achieved fast printing at low cost simply by outputting CMYK values each based on the demanded amounts of a SA solution, $\mathrm{CaCl}_{2}$ solution, and nutrients. In addition, the SCS printer allows a very accurate inoculation of the bacteria onto the medium using accurate overprinting. The CA hydrogel medium had a higher survival rate of bacteria and lower contamination rate compared to PVA hydrogel medium. In summary, we demonstrated that PVA hydrogel and CA hydrogel were an easy-to-use medium for inkjet printing.

\section{Conflict of Interests}

The authors declare that there is no conflict of interests regarding the publication of this paper.

\section{Acknowledgment}

This study was supported by JSPS Grant-in-Aid for Challenging Exploratory Research no. 23658138.

\section{References}

[1] M. Singh, H. M. Haverinen, P. Dhagat, and G. E. Jabbour, "Inkjet printing-process and its applications," Advanced Materials, vol. 22, no. 6, pp. 673-685, 2010.

[2] J. L. Connell, E. T. Ritschdorff, M. Whiteley, and J. B. Shear, “3D printing of microscopic bacterial communities," Proceedings of the National Academy of Sciences of the United States of America, vol. 110, no. 46, pp. 18380-18385, 2013.

[3] T. Xu, W. Zhao, J.-M. Zhu, M. Z. Albanna, J. J. Yoo, and A. Atala, "Complex heterogeneous tissue constructs containing multiple cell types prepared by inkjet printing technology," Biomaterials, vol. 34, no. 1, pp. 130-139, 2013.

[4] W. C. Wilson Jr. and T. Boland, "Cell and organ printing 1: protein and cell printers," Anatomical Record-Part A: Discoveries 
in Molecular, Cellular, and Evolutionary Biology, vol. 272, no. 2, pp. 491-496, 2003.

[5] S. V. Murphy and A. Atala, "3D bioprinting of tissues and organs," Nature Biotechnology, vol. 32, no. 8, pp. 773-785, 2014.

[6] V. Mironov, V. Kasyanov, C. Drake, and R. R. Markwald, "Organ printing: promises and challenges," Regenerative Medicine, vol. 3, no. 1, pp. 93-103, 2008.

[7] S. Moon, S. K. Hasan, Y. S. Song et al., "Layer by layer threedimensional tissue epitaxy by cell-laden hydrogel droplets," Tissue Engineering_Part C: Methods, vol. 16, no. 1, pp. 157-166, 2010.

[8] S. J. Hollister, "Porous scaffold design for tissue engineering," Nature Materials, vol. 4, no. 7, pp. 518-524, 2005.

[9] T. Boland, T. Xu, B. Damon, and X. Cui, "Application of inkjet printing to tissue engineering," Biotechnology Journal, vol. 1, no. 9, pp. 910-917, 2006.

[10] S. Zhang, "Fabrication of novel biomaterials through molecular self-assembly," Nature Biotechnology, vol. 21, no. 10, pp. 11711178, 2003.

[11] B. Modrek and C. Lee, "A genomic view of alternative splicing," Nature Genetics, vol. 30, no. 1, pp. 13-19, 2002.

[12] O. Tadashi, S. Tomohiro, and Y. Nobuko, "Microarray fabrication with covalent attachment of DNA using Bubble Jet technology," Nature Biotechnology, vol. 18, pp. 438-441, 2000.

[13] M. He, O. Stoevesandt, E. A. Palmer, F. Khan, O. Ericsson, and M. J. Taussig, "Printing protein arrays from DNA arrays," Nature Methods, vol. 5, no. 2, pp. 175-177, 2008.

[14] B. Derby, "Bioprinting: Inkjet printing proteins and hybrid cell-containing materials and structures," Journal of Materials Chemistry, vol. 18, no. 47, pp. 5717-5721, 2008.

[15] P. Cooley, D. Wallace, and B. Antohe, "Applications of ink-jet printing technology to BioMEMS and microfluidic systems," Journal of the Association for Laboratory Automation, vol. 7, no. 5, pp. 33-39, 2002.

[16] T. Srimongkon, T. Ishida, K. Igarashi, and T. Enomae, "Development of a bacterial culture system using a paper platform to accommodate media and an ink-jet printing to dispense bacteria," The American Journal of Biochemistry and Biotechnology, vol. 10, no. 1, pp. 81-87, 2014.

[17] J. Elisseeff, "Hydrogels: structure starts to gel," Nature Materials, vol. 7, no. 4, pp. 271-273, 2008.

[18] S. K. H. Gulrez, A. Saphwan, and G. O. Phillips, "Hydrogels: methods of preparation, characterisation and applications," in Progress in Molecular and Environmental Bioengineering-From Analysis and Modeling to Technology Applications, A. Carpi, Ed., 2011.

[19] S. Doyan and A. Doyan, "Dose response and optical properties of dyed poly vinyl alcohol-trichloroacetic acid polymeric blends irradiated with gamma-rays," The American Journal of Applied Sciences, vol. 6, no. 12, pp. 2071-2077, 2009.

[20] E. F. dos Reis, F. S. Campos, A. P. Lage et al., "Synthesis and characterization of poly (vinyl alcohol) hydrogels and hybrids for rMPB70 protein adsorption," Materials Research, vol. 9, no. 2, pp. 185-191, 2006.

[21] Y. K. Yeow, Z. Abbas, K. Khalid, and M. Z. A. Rahman, "Improved dielectric model for polyvinyl alcohol-water hydrogel at microwave frequencies," American Journal of Applied Sciences, vol. 7, no. 2, pp. 270-276, 2010.

[22] J. Ruiz, A. Mantecón, and V. Cádiz, "Synthesis and properties of hydrogels from poly (vinyl alcohol) and ethylenediaminetetraacetic dianhydride," Polymer, vol. 42, no. 15, pp. 6347-6354, 2001.
[23] C. M. Hassan and N. A. Peppas, "Structure and applications of poly (vinyl alcohol) hydrogels produced by conventional crosslinking or by freezing/thawing methods," Advances in Polymer Science, vol. 153, pp. 37-65, 2000.

[24] Encyclopaedia Britannica, Polyvinyl Alcohol (PVA), Encyclopaedia Britannica, 2014, http://www.britannica.com/EBchecked/ topic/469236/polyvinyl-alcohol.

[25] D. W. Jones, E. J. Sutow, and B. S. Graham, "Gel strength and rate of gelation of soft polymers," Dental Materials, vol. 7, no. 2, pp. 138-144, 1991.

[26] J. M. Rosiak and F. Yoshii, "Hydrogels and their medical applications," Nuclear Instruments and Methods in Physics Research, Section B: Beam Interactions with Materials and Atoms, vol. 151, no. 1-4, pp. 56-64, 1999.

[27] A. Blandino, M. Macías, and D. Cantero, "Formation of calcium alginate gel capsules: influence of sodium alginate and $\mathrm{CaCl}_{2}$ concentration on gelation kinetics," Journal of Bioscience and Bioengineering, vol. 88, no. 6, pp. 686-689, 1999.

[28] K. Sultana, G. Godward, N. Reynolds, R. Arumugaswamy, P. Peiris, and K. Kailasapathy, "Encapsulation of probiotic bacteria with alginate-starch and evaluation of survival in simulated gastrointestinal conditions and in yoghurt," International Journal of Food Microbiology, vol. 62, no. 1-2, pp. 47-55, 2000.

[29] L. L. Zhou, D. P. Sun, L. Y. Hu, Y. W. Li, and J. Z. Yang, "Effect of addition of sodium alginate on bacterial cellulose production by Acetobacter xylinum," Journal of Industrial Microbiology \& Biotechnology, vol. 34, no. 7, pp. 483-493, 2007.

[30] L. Wang, R. M. Shelton, P. R. Cooper, M. Lawson, J. T. Triffitt, and J. E. Barralet, "Evaluation of sodium alginate for bone marrow cell tissue engineering," Biomaterials, vol. 24, no. 20, pp. 3475-3481, 2003.

[31] S. Nagakura and M. Gouterman, "Effect of hydrogen bonding on the near ultraviolet absorption of naphthol," The Journal of Chemical Physics, vol. 26, no. 4, pp. 881-886, 1957.

[32] B.-Z. Magnes, D. Pines, N. Strashnikova, and E. Pines, "Hydrogen-bonding interactions of photoacids: correlation of optical solvatochromism with IR absorption spectra," Solid State Ionics, vol. 168, no. 3-4, pp. 225-233, 2004. 

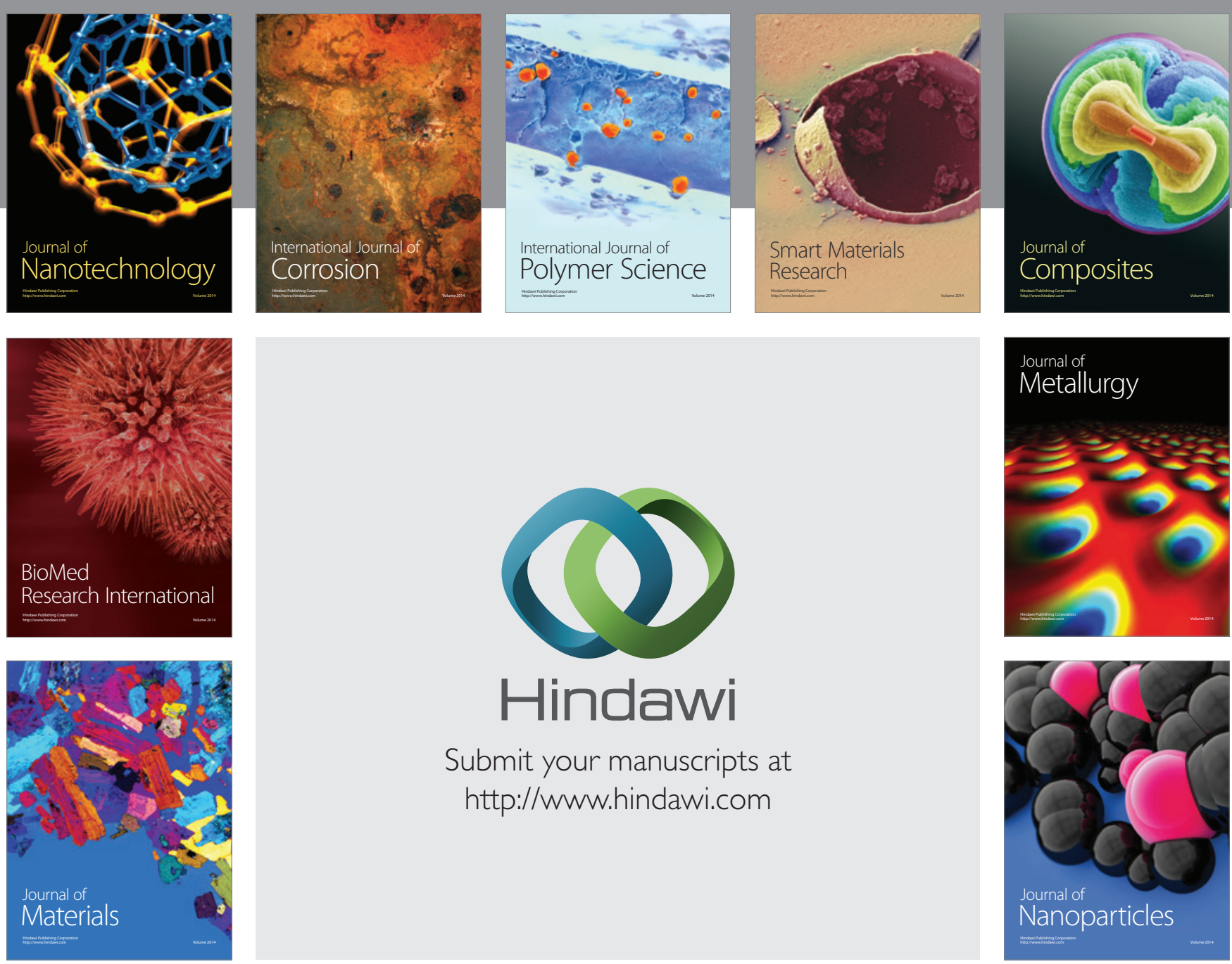

Submit your manuscripts at http://www.hindawi.com
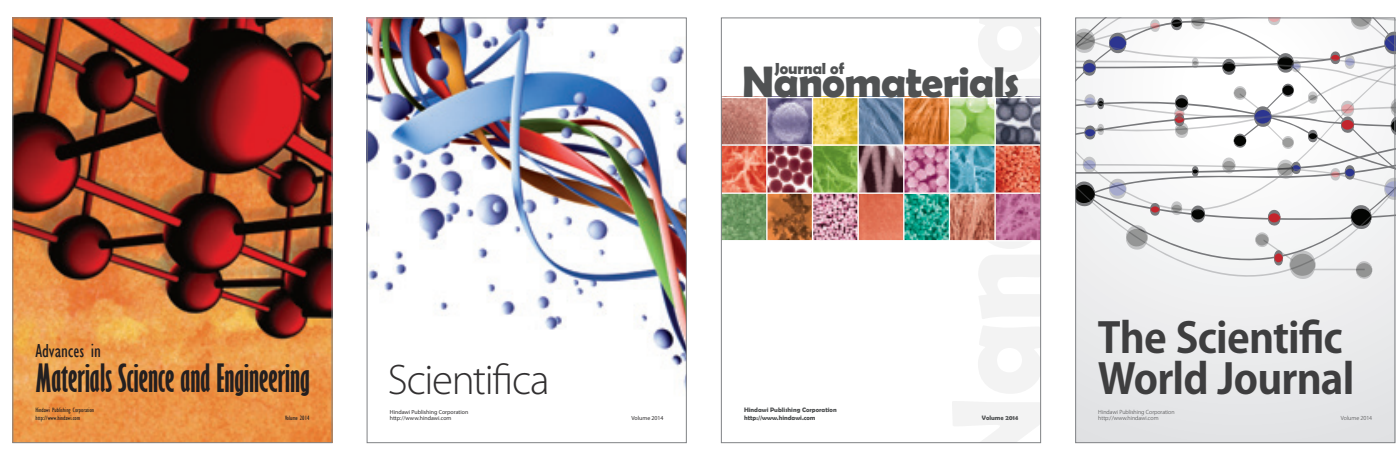

\section{The Scientific World Journal}
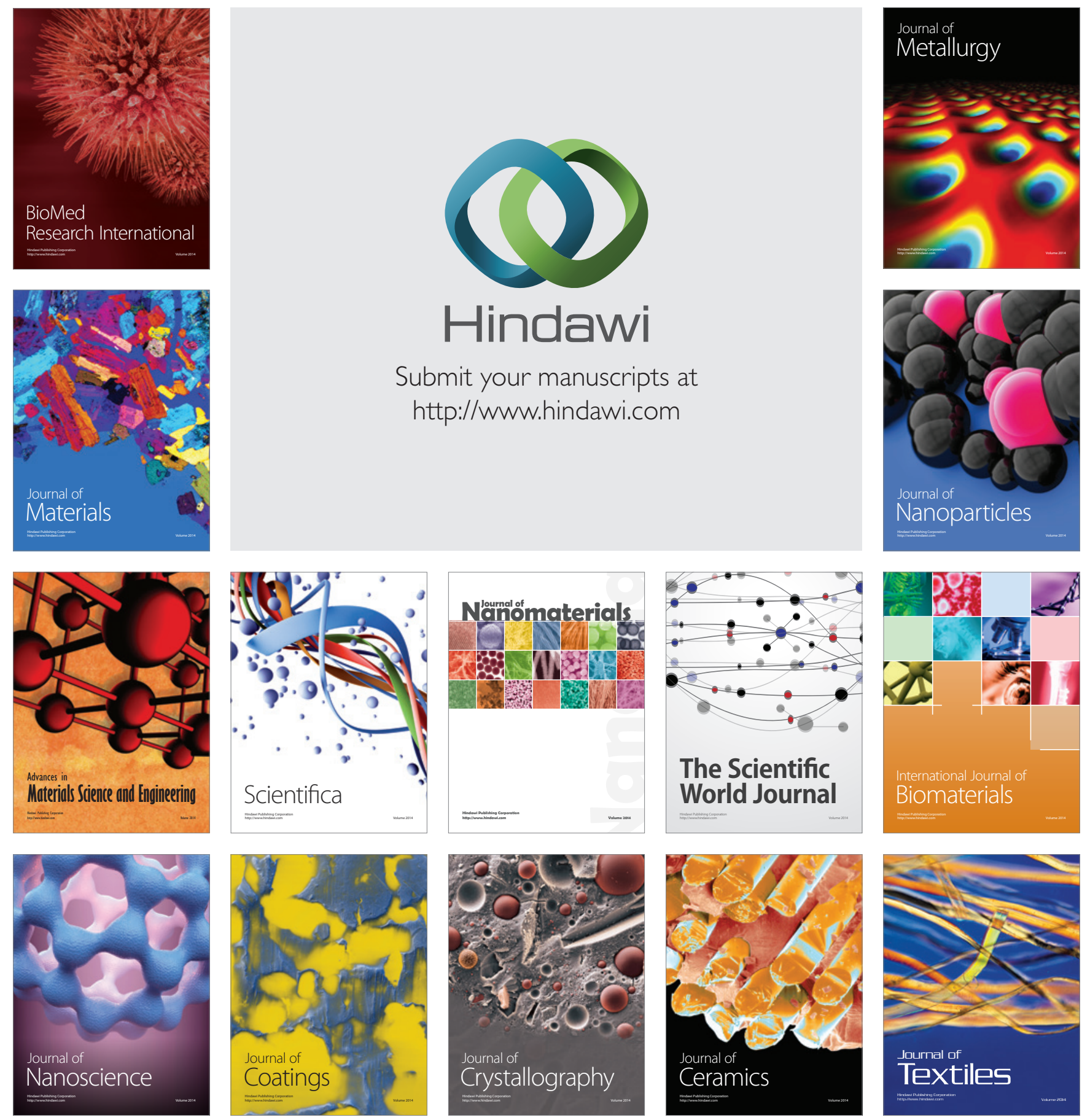